\title{
An Efficiency Management Terminal Considering Demand Response
}

\author{
Liu Yin, Xie Yingxin \\ State Grid Electric Power Research Institute \\ Nanjing, China \\ Beijing GuoDianTong Network Technology Co., Ltd \\ Beijing, China \\ Wang Fengyu \\ State Grid Electric Power Research Institute \\ Nanjing, China \\ Beijing GuoDianTong Network Technology Co., Ltd \\ Beijing, China
}

\author{
Fang Chen \\ Electric Power Research Institute State Grid Shanghai \\ Municipal Electric Power Company \\ Shanghai, China \\ Lei Ting \\ Electric Power Research Institute State Grid Shanghai \\ Municipal Electric Power Company \\ Shanghai, China
}

\begin{abstract}
To support the development of smart grid and demand-side management, this paper designs and implements an efficiency management terminal based on demand response, which can be used to assist users to actively participate in the "Grid - Load" Interaction. First, this paper elaborates the hardware composition of efficiency management terminal. Then realization of diversity load connecting to terminals selfadaptively is given. The four core functions and implementations of the terminal system are described. Finally, a summary is given. The terminal has the advantages of seamless access, simple, high interactivity, etc. And it will play an important role in the development of smart grid.
\end{abstract}

Keywords-efficiency management terminal; protocol adaptation; intelligent control; demand response

\section{INTRODUCTION}

With the deepening of the smart grid and demand response concepts ${ }^{[1-4]}$, the running mode of grid is moving in new directions of two-way interactive, multi-service, intelligent response, energy efficient. Load control device of on the user side is the foundation of learning.

With the higher demanding of electricity service for users, there is need to develop a new class of load control terminal by economic and technological means, which can meet user personalized, differentiated service needs. This is a new challenge for smart grid in the development of intelligent electricity sectors. Reference [5] describes the acquisition and load management terminal based on cloud computing technology, focusing on research in data acquisition and transmission. References [6-8] described in detail the structure and function design of related efficiency management systems. References [9] gave several power hardware design about load management terminal. These studies did not relate to the terminal based on demand response.
Amid all these factors, it is necessary to extend, integrate, or improve the existing grid terminal device, in order to meet information acquisition and control requirements of different underlying devices which come from the upper platform. So this paper designs and implements an efficiency management terminal based on demand response. And it can be used in a new model of efficiency management, which has a lot of characteristics including seamless access of diversity load, flexibility, precision, high degree of user participation.

\section{HARDWARE CONFIGURATION OF EFFICIENCY MANAGEMENT TERMINAL}

This efficiency terminal is simple, rich show, high interactivity, and so on. It offers 12.2 "touch-screen to achieve human-computer interaction. Its hardware configuration is as shown in fig. 1, which mainly has seven modules: central processing unit, memory module, interactive interface module, uplink/downlink communication module, power module and clock module.

(1)Main module of central processing unit: For device access management and intelligent control calculation.

(2)Memory module: It is responsible for storing information about the entire terminal apparatus. The information of load device is including load, power, voltage, power factor and so on. The information of grid is including operating information and price list. The other information is control module parameters, time, and environmental etc.

(3)Interactive interface module: Implement interactive features.

(4) Uplink communication module: It mainly achieves information exchange and communication between this efficiency terminal and the upper master system, through ether net, GPRS, CDMA and other means of communication.

(5) Downlink communication module: It mainly sends data acquisition command to meters and concentrators or sends 
command control to control module of electrical load equipment. It supports a variety of communication RS485, micro-power wireless, PLC (power line carrier), ZigBee, etc.

(6) Power module and clock module provide energy support and time calibration for other modules.

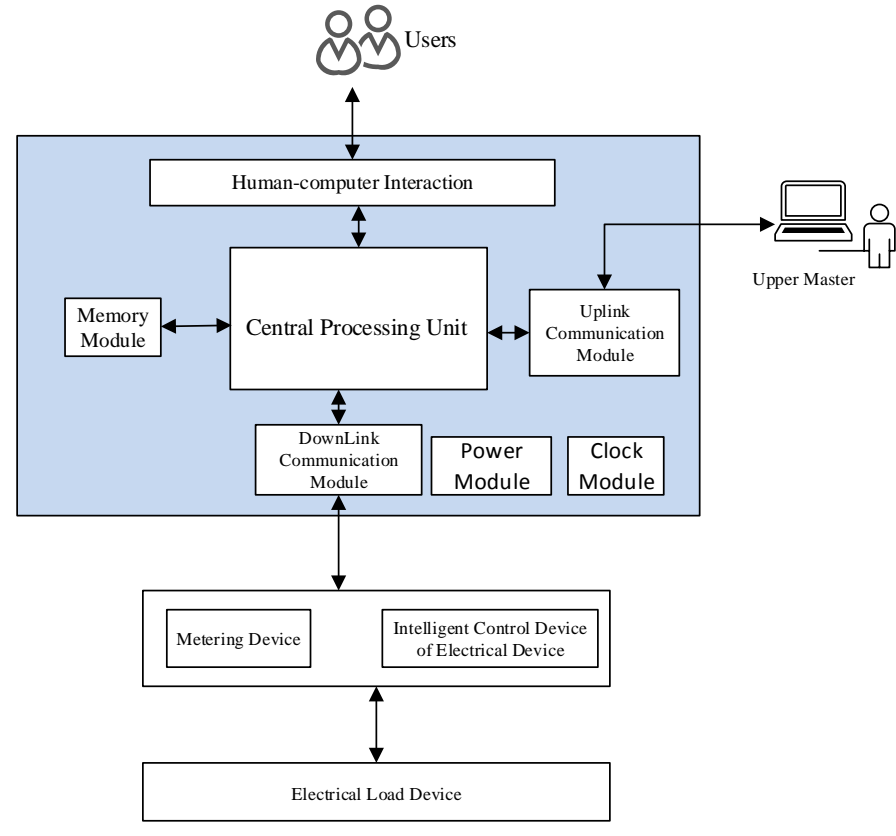

Fig.1 Hardware Configuration of Terminal

\section{IMPLEMENTATIONS OF DEVICE ACCESS TERMINAL}

Due to the growing diversity types of device, such as distributed energy storage, distributed generation devices and intelligent power devices, the interface protocols of equipment and control terminal are various varies. To solve this problem, this terminal is designed an intelligent device interface unit, which mainly achieves protocol adaptation among heterogeneous devices and data acquisition. The specific implementation process is shown in Figure 2.

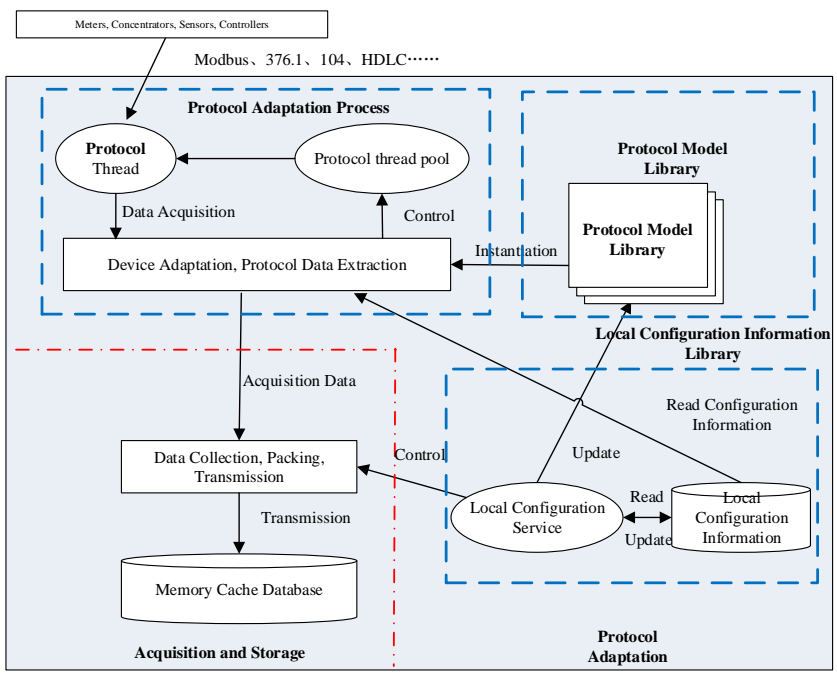

Fig.2 Implementation Process of Device accessing Terminal

\section{A. Protocol Adaptation}

Protocol adapter unit contains protocol model library, local configuration information libraries and protocol adapter. The protocol model library has taken a series of common interface protocol classes, including protocol interface class, protocol implementation class, communication interfaces class, and communication implementation class. These classes encapsulate different protocol frame analysis, assembly operations, and the protocol pool is instantiated and generates protocol. Local configuration information libraries stores the configuration information of meters, concentrators, controllers and other information. This information can be divided into two kinds of structured and unstructured information. Structured information is stored in a small relational database, unstructured information is stored in an XML file. When it comes to protocol adaptation process, since the connected meters, concentrators, controllers does not depend on the channel, after the channel parameter is setted up, the received message is resolved according to the configuration protocol of application-layer. Protocol adaptation is treated by the way of the protocol and channel separation methods. First, the downlink channel adapter, RS485 or some kind of network communications channel is selected and communication parameters is setted. Secondly, the protocol is adapted depending on the application layer protocol, such as 376.1 and so on. The entire protocol adaptation process flow is shown in Figure 3:

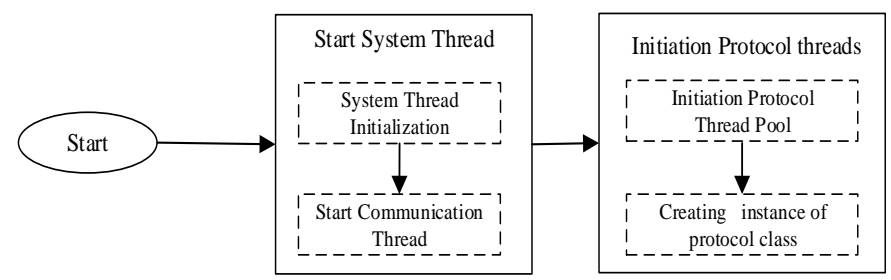

Fig.3 Protocol Adaptation Process Flow

1) System initialization: Reading the global configuration file to get load device name, protocol type, communication ports, IP addresses and other information from the XML file.And protocol class is initialized.

2) Start communication threads: Creating a send and buffer queue, and creating send timer. The system clock checks UDP (User Data Protocol) per second to transmit buffer.Retransmissioning buffer data and updating timer.

3) Initiate protocol pool: Reading load device and its parameters configured by this acquisition point from the global configuration file, uploading to the configuration server Getting load devices and their configuration parameters on the service from the configuration server,and replacing local update XML file. If the communication to and configure server fails,the local configuration will initiate protocol threads.If there is no any configuration information of this acquisition on server-side, the server will record the configuration information.

4) Create protocol class instance: Reading the XML file,creating corresponding protocol class instance. Put it in the initiation queue of the protocol pool,and then sending 
message to protocol class instance to start the thread the load device starts collecting data.

\section{B. Acquisition and Storage}

The main function of the data acquisition and storage is that uploaded data is stored in memory database with a certain format after protocol adaptation. Protocol thread and data acquisition storage exchange data through IPC (Inter-Process Communication). Considering the real-time data on efficiency requirements, binary transfer protocol is adopted. The contents include acquisition point number, item number data, data value, and acquisition time.

\section{FUNCTION DESIGN OF TERMINAL SYSTEM}

This terminal is a low power integrated device which can provide users with collection and control protocol conversion, electricity monitoring and analysis, intelligent control, strategic planning and management etc. It integrates modern communication technology, human-computer interaction and intelligent control technology. According terminal location, this terminal has four functions: power monitoring, power analysis, intelligent control and system setting. Its functional structure is shown in Figure 4.

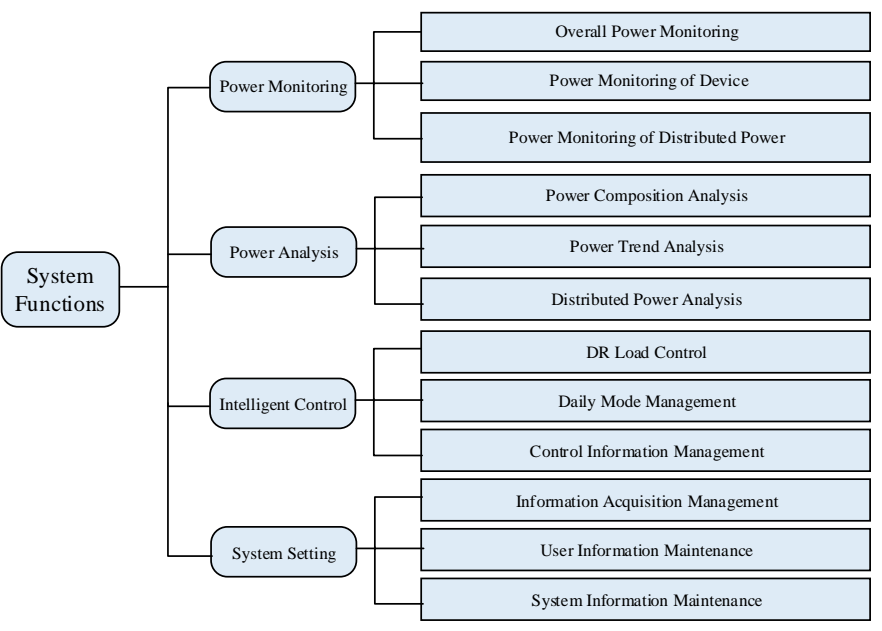

Fig.4 Functional Structure

\section{A. Power Monitoring}

This module mainly have three sub-function modules, including overall power monitoring, power monitoring of device and power monitoring of distributed power, as follows:

1) Overall power monitoring:It monitors the overall power consumption of all devices under the jurisdiction of terminal.

2) Power monitoring of device: It monitors the power consumption of one single device(monitoring points), such as air conditioning, motor and so on.

3) Power monitoring of distributed power: It monitors and displays the relevant parameters of wind, solar and other distributed power systems,including supply power monitoring,distributed power generation status, related electrical parameters of inverter,and environmental parameter monitoring etc.

\section{B. Power Analysis}

Power analysis includes power composition analysis, power trend analysis, and distributed power analysis, as follows:

1) Power composition analysis: It analysis power composition of devices under the jurisdiction of terminal from different angles, and intuitively shows through bar graphs, pie charts and tables.

2) Power trend analysis: It analysis and shows the trend of power, maximum load, power factor, the average price, load factor and other indicators from a variety of analytical dimension. According to the different analytical perspective selected by user, It displays respectively with the corresponding histogram and list display.

3) Distributed power analysis:Through real-time monitoring the SOC status of storage batteries, it analysis external supply power situation of storage batteries.

\section{Intelligent Control}

Intelligent control includes DR load control, daily mode management, control information management.

1) DR load control:It provides the user interface to develop appropriate control events.According to actual needs, the user can set the upper limit of the load within the specified time. System background algorithm automatically generates the appropriate control program on the basis of control constraints and the degree of control priority on the time period, etc. This function consists of two parts, DR event management and DR control event monitor. DR event management includs control event list, add control events, delete control events. DR control event monitor shows the details of selected control events,including control information, control scheme list, control effectiveness and control effectiveness analysis. In addition, the system provides the user the operator interface to quickly locate control events.

2) Daily mode management:It is a functional module which provides users with personalized custom control program. According to their needs, users can set the appropriate control mode.And then the system intelligently control device according to the setting control mode. Users aslo can voluntarily add a new daily pattern, and add a personalized control solutions to this mode. For the selected mode ,starting this mode means that the control scheme under this mode is managed by the terminal,and the system will intelligently control device according to this setting control mode. Ending this mode means that the control scheme under this mode is invalid,and the system will not intelligently control device according to this setting control mode. Also to be noted is that this terminal system can only have one daily pattern which can be enabled at present.

3) Control information management:It provides users with the specified settings controllable load and interactive programs. Users can specify which device is controllable device, according to the load situation of the access terminal. For the users who need to interact with higher-level systems, 
users can develop control programs to interact with the superior system according to their actual situation, and pushes it to the higher-level system, which can provide a basis for decision when the superior system interact with this terminal.

\section{System Setting}

System setting includes three sub-functions: information acquisition management, user information maintenance, system information maintenance.

1) Information acquisition management: It configures the electrical parameters, such as : power, energy, voltage, current, power factor, apparent power, real-time frequency, harmonic voltage ratio, the harmonic current content of harmonic voltage distortion, harmonic current distortion rate, harmonic voltage and harmonic current, etc.

2) User information maintenance: This function is setted up in order to maintain the users who can access to terminal. The permissiona of users are divided into high, medium and low three levels. Users with different levels have different permissions.

3) System information maintenance: It is mainly used to maintain the system information. The device information under the jurisdiction of terminal can be add, view, modify, and delete and other basic operations.The information of electrical parameter points or control points can be setted up. Parameters required for system operation, such as price category, the basic price and control time interval, can be also setted up.

\section{CONCLUSION}

The traditional terminal devices have been unable to meet the needs of the smart grid and demand-side management, it is necessary to study a kind energy efficiency management terminal which emphasizes adaptive access, intelligent interactive, and improving energy efficiency. Based on the above background, this paper designs and implements an efficiency management terminal based on demand response. It describes the implementation of hardware configuration and diversity load seamless access to terminal. Meanwhile it elaborates its four functions including power monitoring, power analysis, intelligent control and system setting. This designed terminal can be well applied to various types of users who have the active participation consciousness of interact with the grid, which is a strong support of smart grid development.

\section{ACKNOWLEDGMENT}

This work was financially supported in part by National Key Technology R\&D Program No.2013BA A01B04.

\section{REFERENCES}

[1] Yu yixin,and Luan wenpeng."Smart grid and its implementations". Proceedings of the CSEE,2009,34:1-8.

[2] Yu daohong, "Status and development of smart grid technology". Electronic Technology \& Software Engineering,2015,01:170.

[3] Cheng wanxing,Shi chankai,and Sunjunping etc."characteristcs and research framwork of automated demand response in smart utilization".Automation of Electric Power System,2013,23:1-7.

[4] Tian shiming,Wang peipei,and Zhang jing."Key Technologies for Demand Response in Smart Grid" .Proceedings of the CSEE, 2014,22:3576-3589.

[5] Wang zhongwen, and Liu zhifang."The Terminal System of Power Data Collection and Management Based on Cloud Computing". China Science and Technology Information,2013,16:95-104.

[6] Zhou ming,and Liu yan."Energy management system in an office building”. Intelligent Building \& City Information. ,2013,03:14-16.

[7] Li jinfeng,Wang zhixin, and Wang liang etc."Research on Control Strategy of Smart Home Efficiency Management System with Household Distributed Photovoltaic Power Station". Electrical and Energy Management Technology,2014,09:52-57.

[8] Dai jinwen,Hujian,and Lin shiming." Intelligent energy management system in demand-side for energy-saving service".Modern Electronics Technique,2012,14:42-45.

[9] Zhao yaling,Meng zhenfei,and Li liyun."Intelligent design of power load control and monitoring system".Electronic Component \& Device Applications, 2010,06:36-39. 